\title{
Blood-Brain Barrier Therapeutics for Neurological Diseases
}

\author{
Aarushi Sahni ${ }^{1}$ and Nicole Katchur" \\ 1Dougherty Valley High School, San Ramon, CA, USA \\ \#Advisor
}

\section{ABSTRACT}

The Blood-Brain Barrier (BBB) is a highly selective filter responsible for allowing certain gases such as oxygen and lipid-soluble molecules to pass (Anand 2014). Its selectiveness makes it challenging for many therapeutics to combat Alzheimer's and Parkinson's disease with external drug therapies. Large-molecule drug therapies never pass the BBB while small-molecule drugs pass only about 5\% of the time (Pardridge 2005). In Alzheimer's disease, tight junctions between endothelial cells degrade, causing an unregulated accumulation of amyloid- $\beta$ (A $\beta$ ) protein (Ramanathan 2015). Consequently, this leads to the formation of neurofibrillary tangles that cut off the nutrient supply to the brain cells and kill neurons (Ramanathan 2015). In Parkinson's disease, astrocyte mutations cause a build-up of $\alpha$-synuclein $(\alpha$ Syn) which affects the neuroinflammatory response and causes dysfunction in dopaminergic neurons (Booth 2017; Meade 2019). New drug therapies for Alzheimer's and Parkinson's continue to undergo trials; some such as FPS-ZM1 and tilavonemab for Alzheimer's and Ravicti for Parkinson's have shown promising results. In addition, similarities in dysfunction for both diseases and some types of cancer have sparked possibilities in retargeting cancer drugs to improve Alzheimer's and Parkinson's pathologies. This review will summarize current therapeutic advancements for Alzheimer's and Parkinson's disease and their possible future contributions.

\section{Introduction}

The blood-brain barrier (BBB) is an intermediary connection between peripheral circulation, responsible for blood distribution from the heart throughout the body, and the central nervous system (CNS) (Kadry 2020). The BBB is semi-permeable, meaning it permits certain gases and small molecules to pass into the brain and protects it from harmful substances (Desai 2007).

\section{Blood-Brain Barrier Location and Structure}

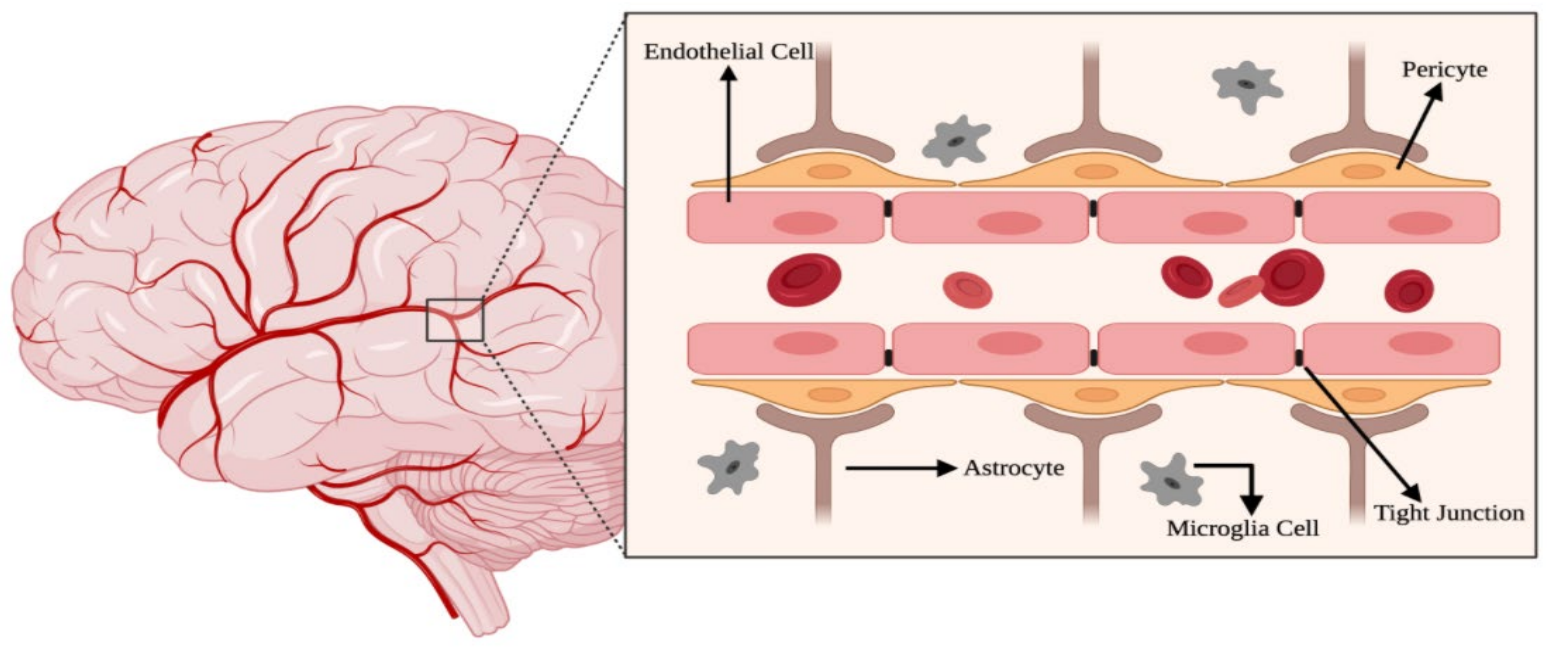


Figure 1. The structure of the blood-brain barrier consists of tight junctions between endothelial cells that filter toxic substances and prevent them from entering the brain [6]. Pericytes and astrocytes protect the endothelial cells and maintain blood flow through the BBB [40]. Microglia cells are immune cells that protect neurons from disease and regulate the inflammatory response (Wake 2011). Created with BioRender.com

The BBB was discovered in 1904 by Paul Ehrlich, a German physician, who conducted an initial experiment by injecting water-soluble dye into the bloodstream of mice (Desai 2007). The dye perfused through all organs in the mice, causing them to stain, excluding the brain (Ribatti 2006). This study was the first experimental evidence indicating there was an existing barrier between the body and brain. Ehrlich's findings built the foundational evidence for future studies proving foreign molecules could not advance through the BBB.

Tight junctions between the endothelial cells of the BBB are directly responsible for the filtration of materials that can pass to the brain (Fig. 1) (Dotiwala 2020). In pathology, neurodegenerative diseases, such as Alzheimer's, are believed to occur when endothelial cells and their tight junction proteins degrade (Lochheard 2020). Consequently, the BBB loses its structure, allowing for external substances to enter the brain, harming neurons, and compromising the function of the brain. Other well-known diseases may also result from mutations in the BBB such as Huntington's and Parkinson's (Monahan 2008; Booth 2017) (Fig. 1).

\section{Methodology}

This review strives to evaluate the hopeful future of developing neurotherapeutics for Parkinson's and Alzheimer's diseases as well as their adverse effects. Primary experiment reports, meta-analysis reviews, and original drug websites were used by an online search in PubMed, Google Scholar, and ClinicalTrials.gov. Articles were then assessed for credibility, relevance, and recency. The search criteria were based on the keywords "blood-brain barrier," "Amyloid$\beta$," "A-synuclein," "Tau phosphorylation," "Alzheimer's disease," "Parkinson's disease," and "neurotherapeutics." Articles that pertained to neuro-diseases unrelated to Alzheimer's and Parkinson's disease were excluded from this study.

\section{Neurotherapeutics and The Blood-Brain Barrier}

Due to the selectiveness of the BBB, only $2 \%$ of small molecules can cross to the brain (Pardridge 2005). This makes it particularly difficult for the growth of effective neurotherapeutic drugs for CNS diseases such as Alzheimer's disease, Parkinson's disease, and epilepsy. About 1 billion people suffer from these neurological diseases and according to the World Health Organization (WHO), it constitutes about $12 \%$ of global deaths yearly (World Health Organization 2006).

Despite the large population of those suffering from CNS diseases, few functional drugs have been designed to combat the dysfunction of the BBB. Large-molecule drugs have been attempted; however, they have not been successful for long-term healing of the barrier. It is a known fact that the suffering rate could diminish with an improvement in the available drug therapeutics for neuro-diseases.

The few small-molecule successes that have been made are only able to treat $5 \%$ of CNS diseases: depression, chronic pain, epilepsy, and schizophrenia (Pardridge 2005). Large-molecule drugs that are combated for neurodegenerative diseases and BBB dysfunction are not able to pass the barrier, so they are treated with therapies that do not directly solve the BBB issue (Pardridge 2005). For instance, patients with Parkinson's disease are given drugs to improve their dopamine levels however no drugs exist to restore the BBB degeneration (Pardridge 2005).

Though the development of BBB therapeutics is severely difficult and understudied, a few pharmaceutical companies are working towards a breakthrough every day. 


\section{Alzheimer's Disease Pathogenesis}

Alzheimer's disease (AD) is the most common dementia in the elderly, resulting in the loss of memory, judgment, cognitive abilities, and reasoning (Bednarcayk \& Lukasiuk 2011). Its multifactorial, complex nature creates difficulties in diagnosis and distinguishing accurate pathogenesis (Drummond \& Wisniewski 2017). The commonly known cause of Alzheimer's is due to neuronal death, however, the role of the BBB dysfunction in the progression of Alzheimer's has been increasingly noticed.

As the BBB's tight junction proteins degrade, it is unable to regulate the clearance of amyloid- $\beta$ (A $\beta)$, a precursor protein found in Alzheimer's, out of the brain (Ramanathan 2015; Upadhaya 2012). The decrease in this regulation causes an accumulation of $\mathrm{A} \beta$. The $\mathrm{A} \beta$ build-up triggers hyperphosphorylation of a protein called tau; the multiple tau proteins form into neurofibrillary tangles that cut off the nerve nutrients transport system, eventually killing the brain cells (Carter \& Lippa 2011).

\section{Amyloid- $\beta$ Oriented Therapeutics}

During the A $\beta$ build-up, ligand-based receptors for advanced glycation end products (RAGE) bind to A $\beta$ more frequently (Angelopoulou 2020). When bound and transported into the brain, these substances contribute heavily to neuroinflammation and neuron death in AD (Anand 2014). To combat this pathogenesis, trial drugs of RAGE antagonists, an opposing receptor ligand that binds to the original receptor and blocks its natural function, were administered (Anand 2014.)

In 2005, azeliragon (PF-04494700 or TTP488) was discovered by Transtech Pharma and was a small small molecule drug directed towards $\mathrm{A} \beta$ in AD (Fig. 2) (Azeliragon 2020). This drug is a non-competitive antagonist that attaches to RAGE; preventing it from binding to proteins such as $A \beta$ and AGEs, in order to prevent its inflammatory response (Azeliragon 2020). Trial I of the drugs was a double-blind study in which this oral antagonist or a placebo was given to 67 randomized patients above the age of 50 for 10 weeks (Sabbagh 2011). The results of the trial within those weeks were positive; subjects had shown an increase in cognitive and functional skills (Sabbagh 2011). However, trial II of the drug for weeks beyond the 10-week mark had shown no long-term changes in meaningful pathogenesis of AD: A $\beta$ levels, inflammation, and cognitive impairment remained close to baseline (Sabbagh 2011). The trials for this drug were abandoned in 2011 (Azeliragon 2020).

But in March of 2013, vTv Therapeutics, formerly known as Transtech Pharma, re-adopted azeliragon (Azeliragon 2020). They continued with a double-blind study of 399 randomized subjects over the age of 50 who were administered doses of azeliragon ( $5 \mathrm{mg} /$ day or $20 \mathrm{mg} /$ day) or placebo for 18 months (Burstein 2014). The study showed a reversible, yet significant, worsening in the deterioration of subjects who were given 20mg/day, however, $5 \mathrm{mg} / \mathrm{day}$ patients showed a significant decrease in both the AD progression trend and A $\beta$ concentration (Burstein 2014). In low doses, the drug is reported to cause gastrointestinal side effects such as diarrhea, constipation, and nausea (Azeliragon 2020). However, the drug was not able to meet its goal of slowing the progression of AD in a study that was recently completed in January of 2021, and its production has been terminated (Fig. 2) (Azeliragon 2020).

More recently a drug called FPS-ZM1 holds considerable value. This high-affinity injected drug was altered from previous attempts beginning around 2012: its molecular weight and hydrogen bonds were decreased, allowing for it to become a small molecule drug (Fig. 2) (Hong 2016). Though it has only been tested in mice so far, its small molecule characteristic allows the antagonist to cross the BBB and bind with RAGE (Hong 2016; Kong 2020). FPSZM1 not only increased cognitive functioning in the mice models but also restored cerebral blood flow and microglial cells (Fig. 1), used to maintain the health of the CNS activity (Kong 2020). Though its mechanisms are not fully understood, the drug has no toxic effects on the animal subject - even with high dosages (Deane 2012). It decreases A $\beta$ concentration, production, and neuroinflammation significantly, and effectively controls neurodegeneration (Kong 
2020). FPS-ZM1 has given the most promising results in the search for an A $\beta$ targeted therapeutic, hopefully pending human clinical trials in the near future.

\section{Tau Phosphorylation Oriented Therapeutics}

Tau phosphorylation plays a significant role in AD, making it another therapeutic target for curing this disease. Tau proteins contribute to microtubule stability, modulating its organization and growth (Anand 2014). However, when $\mathrm{A} \beta$ increases, the tau protein hyperphosphorylation, causing microtubule instability and the presence of tau tangles and neuron death (Carter \& Lippa 2001).

Other contributors to the folding of microtubules are heat shock proteins (HSP), such as HSP 90 or HSP 70 (Campanella 2018; Carter \& Lippa 2021). Some therapeutics meant to decrease tau tangles indirectly focus on HSP inhibition, a similar role to that of antagonists but rather directed towards proteins. To combat the HSP's role in tau tangles, the therapeutic C1206 was created in 2013 as a novel derivative of curcumin - a herbal chemical that has antitumor characteristics (Fig. 2) (Tomeh 2019). C1206 is an administered inhibitor of HSP 90 to patients with leukemia. Test trials show that an increase in C1206 decreases HSP 90 consequentially (Fan 2018). It has also been suggested that curcumin may have the ability to prevent $A \beta$ build-up and tau phosphorylation (Shytle 2021). HSP inhibition has been proven to combat the presence of oxidative stress, a pathology similar in both leukemia and AD, proves potential for retargeting (Luo 2010; Tonnies \& Trushina 2017; Zhang 2018). Though this drug is tested in cancer patients more commonly than AD subjects, the implications of C1206 can be adapted within the AD field, combating similar results for different diseases.

Some discussion on tau targeting has also steered towards tau immunotherapy (Anand 2014). After multiple failures with AD immunotherapy, tau immunotherapy remained promising. In May of 2013, the first tau immunotherapeutic to go through human safety trials was AADvac-1 (Fig. 2) (Brittar 2020). Soon after, the phase II double-blind study consisted of 196 subjects who were administered the immunotherapeutic or a placebo for 24 months (Axon 2020). Results of this study showed a 58\% reduction in the neurodegenerative process and a significant decrease in AD blood biomarkers (Axon 2020). But the more noticeable results lied within the younger AD population; these subjects showed a pronounced change in cognitive and functional skills (Brittar 2020). Side effects that are confirmed to be related to the vaccine include injection site reactions such as warmth, pain, pruritus, and erythema (Novak 2018). AADvac-1 proved a positive future for immunotherapeutics, giving rise to others such as tilavonemab. In 2015, tilavonemab (ABBV-8E12) completed its phase I double-blind study with 30 randomized subjects with worthwhile results and minimal adverse effects (Fig. 2) (West 2017). Phase II trials began in 2019 and are set to conclude in mid2021 (Brittar 2020). 


\section{Alzheimer's and Parkinson's Disease Therapeutics Timeline}

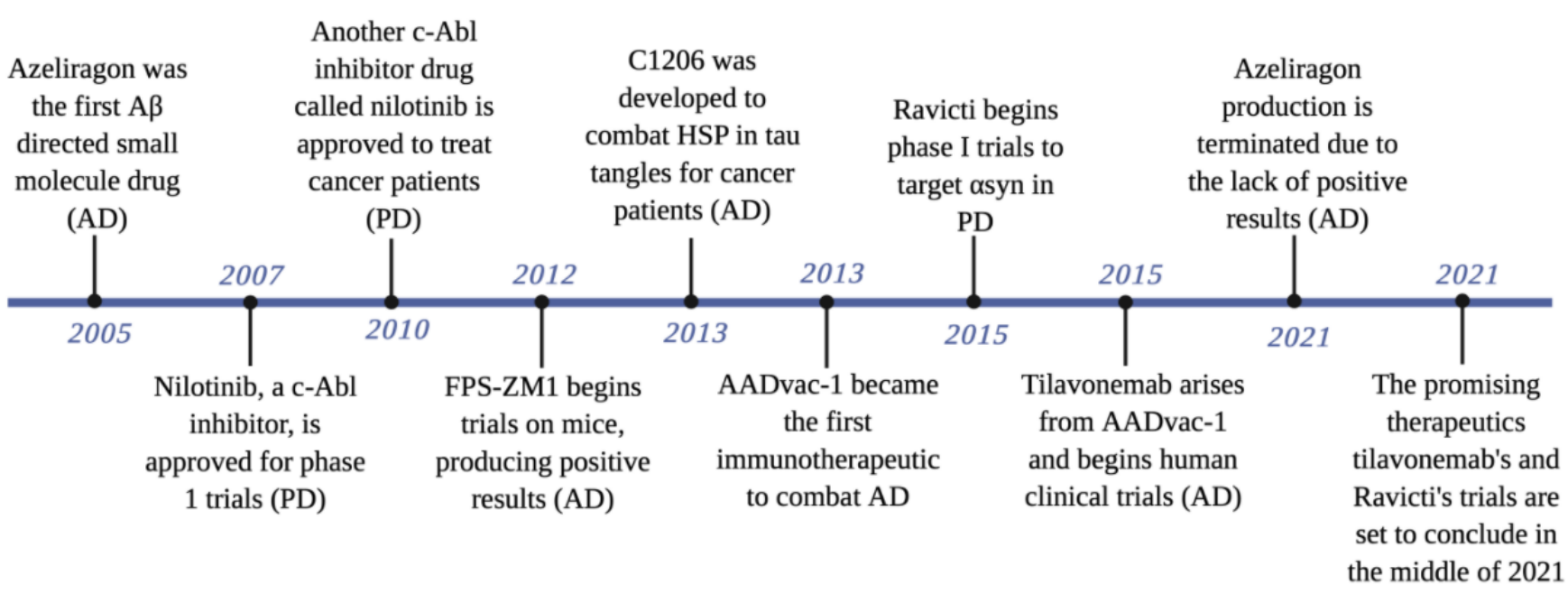

(AD \& PD)

Figure 2. A timeline of current therapeutics for Alzheimer's and Parkinson's disease. Created with BioRender.com

\section{Parkinson's Diseases Pathogenesis}

Parkinson's disease (PD) is the second most common neurodegenerative disorder after AD, characterized by its symptoms of tremors, loss in memory and muscular control, depression, and speech impairments (FEBS Press 2019). Like $\mathrm{AD}, \mathrm{PD}$ is complex and can be induced through genetic, age, or toxic environmental factors, making it difficult to diagnose and cure (FEBS Press 2019). About 10-15\% of PD diagnoses are because of genetic mutations such as PINK1, PARKIN, and DJ-1 (Parkinsons.org 2020). However, no matter the cause, all PD patients experience dopamine, a chemical that controls movement, and nerve loss - which causes an increase in progression of PD symptoms (Cedars 2017).

In the past, many therapeutics have been targeted towards the symptomatic of PD, such as dopamine substitution, and not towards the dysfunctions of the disease (FEBS Press 2019). But recently, there have been more therapeutics tailored to BBB disruption.

Astrocytes around the $\mathrm{BBB}$ are responsible for many parts of neuronal health and brain function (Fig. 1). They protect the endothelial cell tight junction of the BBB from degradation, maintain blood flow and neuron health, and take part in the production of dopamine and cholesterol in the brain (Udovin 2020). Astrocytes also function to clear $\alpha$-synuclein ( $\alpha$ Syn), a protein encoded by the gene SNCA and secreted by neurons (Booth 2017). When $\alpha$ Syn is secreted, the astrocytes take up the toxic protein in order to maintain the health of the brain (Booth 2017). However, when the concentration of $\alpha$ Syn passes the threshold for astrocyte uptake, $\alpha$ Syn aggregates called Lewy bodies begin to form (Meade 2019). Lewy bodies cause a neuroinflammatory response in the brain of PD patients, affect dopaminergic neurons, and decrease BBB integrity; all pathologies in PD (Booth 2017).

\section{A-Synuclein Oriented Therapeutics}


In PD pathology there are many other contributing factors to Lewy body accumulation. The $\alpha$ Syn aggregates trigger a response in Abelson tyrosine kinase (c-Abl), resulting in its activation (Karim 2020). c-Abl activation causes dopaminergic neuron death and further accumulation of $\alpha$ Syn in the brain (Karim 2020).

In 2007, a c-Abl inhibitor called Tasigna (AMN107 or nilotinib) was approved for phase 1 human trials in PD patients (Fig. 2) (Nilotinib 2009). Tasigna was previously a drug tailored to c-Abl activation in patients with blood cancers, such as leukemia, but has been proceeding through clinical trials to combat the same effect in PD (Deepa Dash 2019). After demonstrating positive results in phase I trials, phase II trials were completed in 2019 with 75 randomized patients to test the safety and tolerability of this oral drug (National Society 2020; Pagan 2020). The participants were given $150 \mathrm{mg} /$ day, 300mg/day, or a placebo for 12 months in this double-blind study (National Society 2020). During the trial there were some adverse effects like headaches, cardiovascular severe adverse events, and stroke; however, it is unclear if these were related to the drug or pre-existing factors (Pagan 2020). The drug was still considered to be safe and able to be detected in cerebrospinal fluid (CSF), which circulates material through the blood and brain (Pagan 2020). Though not many positive results were seen in the 2019 study, there is hope for the phase III trials to produce the anticipated promising outcome (Pagan 2020).

Another c-Abl inhibitor in 2010, bafetinib (INNO-406), proved to reach a CSF concentration much higher than that of Tasigna (Fig.2) (Brahmachari 2017). This oral therapeutic has not been tested in PD disease patients, but much like Tasigna is used to treat types of cancer as well. Because of the similarities in mechanism, there is a possible discussion of testing bafetinib in PD for more promising results (Brahmachari 2017).

In 2015 a therapeutic called Ravicti (Glycerol Phenylbutyrate) was discovered, however, its phase I trials began years later (Fig. 2). Ravicti is currently going through its phase I study with 20 non-randomized participants in order to evaluate the consistency of effects on PD (ClinicalTrials.gov 2021). Ravicti turns on a protective gene in the brain that allows for the $\alpha$ Syn build-up to be released into the blood where it can be eliminated (Zhou 2013). The patients in this study are given 20 grams of Ravicti a day in liquid form for 21 days (ClinicalTrials.gov 2021) and tested for increased levels of $\alpha$ Syn in the bloodstream (ClinicalTrials.gov 2021). No adverse effects or results have been reported yet as the study is set to complete in April of 2021.

\section{Future of Parkinson's Disease Therapeutics}

Another large discussion topic for Parkinson's disease is in the DJ-1 gene encoded by the PARK7 gene (Booth 2017). PARK7 has been reported to be expressed in high levels in astrocytes, further solidifying a connection between the DJ-1 gene and astrocytes, specifically lipid rafts created by astrocytes (Booth 2017). Lipid rafts are cholesterol-enriched microdomains in the brain that play a role in the immune and inflammatory response in PD (Booth 2017). Cholesterol is a lipid that is typically made in the liver; however, the BBB does not permit the regulation of this cholesterol, causing the brain to create its own through astrocytes (Ferris 2017). This demonstrates a connection between astrocytes and cholesterol and the generation of lipid rafts in the brain. It has been shown that the PARK7 mutation causes increased degradation of the lipid raft proteins flotillin-1 and caveolin-1, leading to lipid raft disassembly and inflammation in the brain (Booth 2017). Additionally, there have been studies demonstrating that there is a depletion of cholesterol in PD brains (Jin 2019).

Current discussion for PD therapeutics has leaned towards targeting flotillin-1 and caveolin-1 proteins in lipid rafts and other genes involved in its degradation (Kang 2018). There have not been lipid raft-targeting therapeutics specifically for PD as there is still much to learn about the factors of lipid raft dysfunction (Kang 2018). However, recent therapies have been tested for lipid raft dysfunction and demonstrates possible future research on PD brains and cholesterol substitutions in the brain (Sanchez-Wandelmer 2009; Sviridov 2020).

\section{Discussion}


The blood-brain barrier dysfunction of Alzheimer's and Parkinson's disease have been increasingly targeted for therapeutic purposes. In AD, FPS-ZM1 has the greatest potential as a future therapeutic because it produces a positive improvement in mouse models with no adverse effects. There is a rising discussion of the implementation of FPSZM1 in human trials, hopefully portraying similar outcomes to the mouse models in A $\beta$ breakdown. Immunotherapy has also been showing a positive shift from small-molecule drugs. Immunotherapy treatments act on the immune system of the patient, rather than the site of dysfunction itself. This can allow for the immune response to function more efficiently and stop the further progression of $\mathrm{AD}$ before symptoms become severe.

In addition to these, there is a possibility of using HSP 90 inhibitors targeted to cancer in AD patients. The similar mechanism of the HSP 90 allows for future use of C1206 in AD to combat tau phosphorylation and A $\beta$ buildup.

In PD there is also a possibility of using cancer-targeted drugs such as Tasigna and bafetinib. Using these drugs and implementing them into another disease would allow for greater variability in already approved and developed drugs. On average, only 5 in 5,000 newly developed drugs are approved by the FDA to proceed through preclinical trials, which take about three and a half years (Medicine Net 2019). The odds of a new drug for Alzheimer's and Parkinson's being approved is drastically low, and many times these drugs can be thrown out for lack of promising results or unsafe symptoms. Using cancer drugs that have been approved and have passed preclinical trials would allow for promising drugs to reach the market more quickly, possibly saving thousands of people. This would also save millions of dollars which may be used to fund new drug discoveries. Retargeting cancer drugs that have such similar mechanisms is the fastest and most promising future of AD and PD drug therapies substitutions in the brain (Sanchez-Wandelmer 2009; Sviridov 2020).

\section{Limitations}

The limitations of this study stem from the uncertainties of how Alzheimer's and Parkinson's disease begin to develop. There is still much unknown about the origins of the diseases and other factors. Though there are many pathogenesis that contribute to the progression of the diseases, it is unclear if the dysfunctions the current therapeutics treat will most effectively decrease its advancements. Because this study is not an exhaustive review on the technicalities of retargeting drugs, the procedure for this course of action is unclear.

\section{Conclusion}

The understanding of the relationship between the blood-brain barrier and the pathogenesis of Alzheimer's and Parkinson's disease is expanding. Because little is understood about the genesis of these diseases, neurology research is continuing to modify how therapeutics are administered and the dysfunctions they target. These therapeutic drugs are advancing to target dysfunction in amyloid- $\beta$ and tau phosphorylation in AD, and $\alpha$-synuclein in PD. Currently there is no cure for neurodegenerative diseases but new medications are undergoing clinical trials in hopes of decreasing the progression of degeneration.

Although $\mathrm{AD}$ and $\mathrm{PD}$ are neurodegenerative diseases concerned with neuron and BBB dysfunction in the brain, seemingly having no similitude to cancer, there is prospect of retargeting drugs used to treat types of cancer. Upon further research, many successfully developed drugs specifically for leukemia have similar mechanisms of actions. Using these drugs to treat $\mathrm{AD}$ and $\mathrm{PD}$ as well can allow for new discoveries within the neurology and oncology fields, possibly progressing to a cure for both diseases more quickly. It is clear that this painful disease is becoming a large issue and a way of treating or managing the dysfunctions is essential. There is still much to learn about the effectiveness of utilizing cancer drugs for AD and PD, but combined with the current therapeutics in clinical trials there is more confidence in lessening the progression of these widespread diseases. 


\section{Acknowledgements}

I would like to thank Nicole Katchur for her guidance, encouragement, and edits throughout the writing and research process of this review.

\section{References}

Anand, R., Gill, K. D., \& Mahdi, A. A. (2014). Therapeutics of Alzheimer's disease: Past, present and future. Neuropharmacology, 76 Pt A, 27-50.

Angelopoulou, E., Paudel, Y. N., Shaikh, M. F., \& Piperi, C. (2020). Flotillin: A Promising Biomarker for Alzheimer's Disease. Journal of Personalized Medicine, 10(2). https://doi.org/10.3390/jpm10020020

Axon Presented Positive Phase II Trial Results of AADvac1 at AAT-AD/PD 2020. (n.d.). Retrieved January 25, 2021, from https://www.biospace.com/article/axon-presented-positive-phase-ii-trial-results-of-aadvac1-at-aat-ad-pd$2020 /$

Azeliragon. (n.d.). Retrieved January 28, 2021, from https://www.alzforum.org/therapeutics/azeliragon

Bednarczyk, J., \& Lukasiuk, K. (2011). Tight junctions in neurological diseases. Acta Neurobiologiae Experimentalis, 71(4), 393-408.

Bittar, A., Bhatt, N., \& Kayed, R. (2020). Advances and considerations in AD tau-targeted immunotherapy. Neurobiology of Disease, 134, 104707.

Booth, H. D. E., Hirst, W. D., \& Wade-Martins, R. (2017). The Role of Astrocyte Dysfunction in Parkinson's Disease Pathogenesis. Trends in Neurosciences, 40(6), 358.

Brahmachari, S., Karuppagounder, S. S., Ge, P., Lee, S., Dawson, V. L., Dawson, T. M., \& Ko, H. S. (2017). c-Abl and Parkinson's Disease: Mechanisms and Therapeutic Potential. In Journal of Parkinson's Disease (Vol. 7, Issue 4, pp. 589-601). https://doi.org/10.3233/jpd-171191

Burstein, A. H., Grimes, I., Galasko, D. R., Aisen, P. S., Sabbagh, M., \& Mjalli, A. M. M. (2014). Effect of TTP488 in patients with mild to moderate Alzheimer's disease. BMC Neurology, 14, 12.

Campanella, C., Pace, A., Caruso Bavisotto, C., Marzullo, P., Marino Gammazza, A., Buscemi, S., \& Palumbo Piccionello, A. (2018). Heat Shock Proteins in Alzheimer's Disease: Role and Targeting. International Journal of Molecular Sciences, 19(9). https://doi.org/10.3390/ijms19092603

Carter, J., \& Lippa, C. F. (2001). Beta-amyloid, neuronal death and Alzheimer's disease. Current Molecular Medicine, 1(6), 733-737.

Cerebrospinal Fluid (CSF). (n.d.). Retrieved February 26, 2021, from https://www.nationalmssociety.org/Symptoms-Diagnosis/Diagnosing-Tools/Cerebrospinal-Fluid-(CSF) 
Deane, R., Singh, I., Sagare, A. P., Bell, R. D., Ross, N. T., LaRue, B., Love, R., Perry, S., Paquette, N., Deane, R. J., Thiyagarajan, M., Zarcone, T., Fritz, G., Friedman, A. E., Miller, B. L., \& Zlokovic, B. V. (2012). A multimodal RAGE-specific inhibitor reduces amyloid $\beta$-mediated brain disorder in a mouse model of Alzheimer disease. The Journal of Clinical Investigation, 122(4), 1377.

Deepa Dash, V. G. (2019). Anticancer Drugs for Parkinson's Disease: Is It a Ray of Hope or Only Hype? Annals of Indian Academy of Neurology, 22(1), 13.

Desai, B. S., Monahan, A. J., Carvey, P. M., \& Hendey, B. (2007). Blood-brain barrier pathology in Alzheimer's and Parkinson's disease: implications for drug therapy. Cell Transplantation, 16(3), 285-299.

Dotiwala, A. K., McCausland, C., \& Samra, N. S. (2020). Anatomy, Head and Neck, Blood Brain Barrier. In StatPearls. StatPearls Publishing.

Drug Approvals - From Invention to Market...12 Years! (n.d.). Retrieved April 25, 2021, from https://www.medicinenet.com/script/main/art.asp?articlekey=9877

Drummond, E., \& Wisniewski, T. (2017). Alzheimer's disease: experimental models and reality. Acta Neuropathologica, 133(2), 155-175.

Exploring the Link Between Dopamine and Parkinson's Disease. (n.d.). Retrieved February 25, 2021, from https:/www.cedars-sinai.org/blog/exploring-the-link-between-dopamine-and-parkinsons-disease.html

Fan, Y.-J., Zhou, Y.-X., Zhang, L.-R., Lin, Q.-F., Gao, P.-Z., Cai, F., Zhu, L.-P., Liu, B., \& Xu, J.-H. (2018). C1206, a novel curcumin derivative, potently inhibits Hsp90 and human chronic myeloid leukemia cells in vitro. Acta Pharmacologica Sinica, 39(4), 649.

FEBS Press. (n.d.). Retrieved February 25, 2021, from https://febs.onlinelibrary.wiley.com/doi/full/10.1111/febs.12335

Ferris, H. A., Perry, R. J., Moreira, G. V., Shulman, G. I., Horton, J. D., \& Kahn, C. R. (2017). Loss of astrocyte cholesterol synthesis disrupts neuronal function and alters whole-body metabolism. Proceedings of the National Academy of Sciences of the United States of America, 114(5), 1189-1194.

Genetics and Parkinson's. (n.d.). Retrieved February 25, 2021, from https://www.parkinson.org/understandingparkinsons/causes/genetics

Hong, Y., Shen, C., Yin, Q., Sun, M., Ma, Y., \& Liu, X. (2016). Effects of RAGE-Specific Inhibitor FPS-ZM1 on Amyloid- $\beta$ Metabolism and AGEs-Induced Inflammation and Oxidative Stress in Rat Hippocampus. Neurochemical Research, 41(5), 1192-1199.

Jin, U., Park, S. J., \& Park, S. M. (2019). Cholesterol Metabolism in the Brain and Its Association with Parkinson's Disease. 28(5), 554-567.

Kadry, H., Noorani, B., \& Cucullo, L. (2020). A blood-brain barrier overview on structure, function, impairment, and biomarkers of integrity. Fluids and Barriers of the CNS, 17(1), 69. 
Kang, S.-J., Kim, J. S., \& Park, A. S. M. (2018). Ubiquitin C-terminal Hydrolase L1 Regulates Lipid Raft-dependent Endocytosis. Experimental Neurobiology, 27(5), 377-386.

Karim, M. R., Liao, E. E., Kim, J., Meints, J., Martinez, H. M., Pletnikova, O., Troncoso, J. C., \& Lee, M. K. (2020). $\alpha$-Synucleinopathy associated c-Abl activation causes p53-dependent autophagy impairment. Molecular Neurodegeneration, 15(1), 1-18.

Kong, Y., Liu, C., Zhou, Y., Qi, J., Zhang, C., Sun, B., Wang, J., \& Guan, Y. (2020). Progress of RAGE Molecular Imaging in Alzheimer's Disease. Frontiers in Aging Neuroscience, 12, 227.

Lochhead, J. J., Yang, J., Ronaldson, P. T., \& Davis, T. P. (2020). Structure, Function, and Regulation of the BloodBrain Barrier Tight Junction in Central Nervous System Disorders. Frontiers in Physiology, $11,914$.

Luo, W., Sun, W., Taldone, T., Rodina, A., \& Chiosis, G. (2010). Heat shock protein 90 in neurodegenerative diseases. In Molecular Neurodegeneration (Vol. 5, Issue 1, p. 24). https://doi.org/10.1186/1750-1326-5-24

Meade, R. M., Fairlie, D. P., \& Mason, J. M. (2019). Alpha-synuclein structure and Parkinson's disease - lessons and emerging principles. Molecular Neurodegeneration, 14(1), 1-14.

Monahan, A. J., Warren, M., \& Carvey, P. M. (2008). Neuroinflammation and peripheral immune infiltration in Parkinson's disease: an autoimmune hypothesis. Cell Transplantation, 17(4), 363-372.

Nilotinib. (n.d.). Retrieved February 25, 2021, from

https://www.sciencedirect.com/topics/neuroscience/nilotinib\#: :text=Nilotinib $\% 2 \mathrm{C} \% 20 \mathrm{a} \% 20$ second $\% 2 \mathrm{Dgeneration}$ \%20TKI,CML\%20\%5B44\%E2\%80\%9346\%5D.

[No title]. (n.d.). Retrieved February 23, 2021, from

https://www.alzdiscovery.org/uploads/cognitive_vitality_media/Azeliragon-Cognitive-Vitality-For-Researchers.pdf

Novak, P., Schmidt, R., Kontsekova, E., Kovacech, B., Smolek, T., Katina, S., Fialova, L., Prcina, M., Parrak, V., Dal-Bianco, P., Brunner, M., Staffen, W., Rainer, M., Ondrus, M., Ropele, S., Smisek, M., Sivak, R., Zilka, N., Winblad, B., \& Novak, M. (2018). FUNDAMANT: an interventional 72-week phase 1 follow-up study of AADvac1, an active immunotherapy against tau protein pathology in Alzheimer's disease. Alzheimer's Research \& Therapy, 10(1), 1-16.

Pagan, F. L., Hebron, M. L., Wilmarth, B., Torres-Yaghi, Y., Lawler, A., Mundel, E. E., Yusuf, N., Starr, N. J., Anjum, M., Arellano, J., Howard, H. H., Shi, W., Mulki, S., Kurd-Misto, T., Matar, S., Liu, X., Ahn, J., \& Moussa, C. (2020). Nilotinib Effects on Safety, Tolerability, and Potential Biomarkers in Parkinson Disease: A Phase 2 Randomized Clinical Trial. JAMA Neurology, 77(3), 309.

Pardridge, W. M. (2005). The blood-brain barrier: bottleneck in brain drug development. NeuroRx: The Journal of the American Society for Experimental NeuroTherapeutics, 2(1), 3-14.

Phenylbutyrate for Alpha-synuclein Clearance from the Brain. (n.d.). Retrieved February 26, 2021, from https://www.michaeljfox.org/grant/phenylbutyrate-alpha-synuclein-clearance-brain 
Phenylbutyrate Response as a Biomarker for Alpha-synuclein Clearance From the Brain. (n.d.). Retrieved February 26, 2021, from https://clinicaltrials.gov/ct2/show/NCT02046434

Ramanathan, A., Nelson, A. R., Sagare, A. P., \& Zlokovic, B. V. (2015). Impaired vascular-mediated clearance of brain amyloid beta in Alzheimer's disease: the role, regulation and restoration of LRP1. In Frontiers in Aging Neuroscience (Vol. 7). https://doi.org/10.3389/fnagi.2015.00136

Ribatti, D., Nico, B., Crivellato, E., \& Artico, M. (2006). Development of the blood-brain barrier: a historical point of view. Anatomical Record. Part B, New Anatomist, 289(1), 3-8.

Sabbagh, M. N., Agro, A., Bell, J., Aisen, P. S., Schweizer, E., \& Galasko, D. (2011). PF-04494700, an oral inhibitor of receptor for advanced glycation end products (RAGE), in Alzheimer disease. Alzheimer Disease and Associated Disorders, 25(3), 206-212.

Sánchez-Wandelmer, J., Dávalos, A., Herrera, E., Giera, M., Cano, S., de la Peña, G., Lasunción, M. A., \& Busto, R. (2009). Inhibition of cholesterol biosynthesis disrupts lipid raft/caveolae and affects insulin receptor activation in 3T3-L1 preadipocytes. Biochimica et Biophysica Acta, 1788(9). https://doi.org/10.1016/j.bbamem.2009.05.002

Shytle, R. D., Tan, J., Bickford, P. C., Rezai-Zadeh, K., Hou, L., Zeng, J., Sanberg, P. R., Sanberg, C. D., Alberte, R. S., Fink, R. C., \& Roschek, B., Jr. (2012). Optimized turmeric extract reduces $\beta$-Amyloid and phosphorylated Tau protein burden in Alzheimer's transgenic mice. Current Alzheimer Research, 9(4), 500-506.

Sviridov, D., Miller, Y. I., Ballout, R. A., Remaley, A. T., \& Bukrinsky, M. (2020). Targeting Lipid Rafts-A Potential Therapy for COVID-19. In Frontiers in Immunology (Vol. 11). https://doi.org/10.3389/fimmu.2020.574508

Tomeh, M. A., Hadianamrei, R., \& Zhao, X. (2019). A Review of Curcumin and Its Derivatives as Anticancer Agents. International Journal of Molecular Sciences, 20(5). https://doi.org/10.3390/ijms20051033

Tönnies, E., \& Trushina, E. (2017). Oxidative Stress, Synaptic Dysfunction, and Alzheimer's Disease. In Journal of Alzheimer's Disease (Vol. 57, Issue 4, pp. 1105-1121). https://doi.org/10.3233/jad-161088

Udovin, L., Quarracino, C., Herrera, M. I., Capani, F., Otero-Losada, M., \& Perez-Lloret, S. (2020). Role of Astrocytic Dysfunction in the Pathogenesis of Parkinson's Disease Animal Models from a Molecular Signaling Perspective. In Neural Plasticity (Vol. 2020, pp. 1-10). https://doi.org/10.1155/2020/1859431

Upadhaya, A. R. (2012). Analysis of Amyloid Beta (A $\beta$ ) Protein in Amyloid Precursor Protein (APP) Transgenic Mouse Models of Alzheimer's Disease (AD) and in Human Brains.

Wake, H., Moorhouse, A. J., \& Nabekura, J. (2011). Functions of microglia in the central nervous system--beyond the immune response. Neuron Glia Biology, 7(1), 47-53.

Website. (n.d.). Retrieved February 25, 2021, from Azeliragon. [cited 28 Jan 2021]. Available: https://www.alzforum.org/therapeutics/azeliragon

West, T., Hu, Y., Verghese, P. B., Bateman, R. J., Braunstein, J. B., Fogelman, I., Budur, K., Florian, H., Mendonca, N., \& Holtzman, D. M. (2017). Preclinical and Clinical Development of ABBV-8E12, a Humanized Anti-Tau 
Antibody, for Treatment of Alzheimer's Disease and Other Tauopathies. The Journal of Prevention of Alzheimer's Disease, 4(4). https://doi.org/10.14283/jpad.2017.36

World Health Organization. (2006). Neurological Disorders: Public Health Challenges. World Health Organization.

Zhang, J., Lei, W., Chen, X., Wang, S., \& Qian, W. (2018). Oxidative stress response induced by chemotherapy in leukemia treatment (Review). In Molecular and Clinical Oncology. https://doi.org/10.3892/mco.2018.1549 